\title{
Sinonasal Teratocarcinosarcoma: An Underdiagnosed Entity Posing Diagnostic Challenges
}

\author{
Mukund SABLE${ }^{1}$, Aanchal KAKKAR ${ }^{1}$, Kanwaljeet GARG ${ }^{2}$, Vaishali SURI ${ }^{1}$ \\ ${ }^{1}$ All India Institute of Medical Sciences, Department of Pathology, New Delhi, India \\ ${ }^{2}$ All India Institute of Medical Sciences, Department of Neurosurgery, New Delhi, India
}

\section{ABSTRACT}

Sinonasal teratocarcinosarcoma (SNTCS) is a rare malignancy of the nasal cavity and paranasal sinuses. Histologically, this tumor consists of mature and immature components of epithelial, mesenchymal and neuroectodermal origin, which may be present in varying proportions. This morphological heterogeneity often leads to misdiagnosis, particularly in biopsies. These patients have dismal prognosis, with frequent recurrences and short mean survival periods. Due to its rarity, clinical characteristics and optimal therapy are not well-characterized. We report a case of SNTCS with intracranial extension, which was treated with surgical resection, followed by radiotherapy. Knowledge of this entity is necessary for accurate diagnosis and proper management.

KEYWORDS: Sinonasal, Teratocarcinosarcoma, Neuroectodermal tumor, Histopathology

\section{INTRODUCTION}

S inonasal teratocarcinosarcoma (SNTCS) is an extremely uncommon malignant neoplasm with aggressive behavior, poor prognosis, high recurrence rate and short median survival period (3). It poses many diagnostic challenges due to its rarity, morphological complexity and lack of awareness. This neoplasm was first described by Shanmugaratnam in 1983 as a carcinosarcoma (5). A year later, Heffner and Hyams gave it the nomenclature of 'teratocarcinosarcoma' and it was included as a distinct entity in the 2005 edition of WHO Pathology and Genetics of Head and Neck tumors (1-3). To the best of our knowledge, 86 cases have been reported to date. We report a case of SNTCS of the nasopharynx with intracranial extension into the anterior cranial fossa, which emphasis on diagnostic challenges, histogenesis and treatment options.

\section{CASE REPORT}

A 26-year-old man presented with complaints of progressive nasal obstruction and intermittent epistaxis for six months, followed by right eye proptosis for six weeks. Clinically, the patient was conscious but disoriented, with a Glasgow Coma Scale score of E4V4M6. On inspection of the nasal cavities, an erythematous, fleshy mass was identified on the right side. On neurological examination, sense of smell was decreased on both sides. Ocular examination revealed proptosis of the right eye with restricted extra-ocular movements. Visual acuity was normal. Fundus examination revealed papilledema on the right side. No other cranial nerve deficits were noted.

Magnetic resonance (MR) imaging showed a midline heterogenous lesion measuring $7.4 \mathrm{~cm}$ horizontally and $7.0 \mathrm{~cm}$ anterio-posteriorly, involving bilateral nasal cavities, right ethmoid sinus and the nasopharynx, with extension into the right orbit and anterior cranial fossa. The mass was hypointense on T1and isointense on T2- weighted images (Figure 1A-F).

The patient underwent bi-frontal orbital craniotomy. The intradural portion of tumor involving frontal lobe was excised completely; however, the portion extending into the posterior ethmoid sinuses could not be resected. Near-total excision of the tumor with anterior cranial fossa repair was done. The postoperative recovery period was uneventful. Post-operative 
imaging showed residual tumor within the ethmoid sinuses. MR images taken a month later showed increase in size of the residual tumor. The patient was scheduled to receive radiotherapy. Meanwhile, he presented to the Emergency Department with intractable seizures, following which he expired.

Histopathological examination revealed a neuroectodermal tumor, which comprised of islands and nests of small round blue cells in a myxoid stroma composed of spindle shaped cells. Focally, the primitive round cells formed true rosettes (Figures $2 \mathrm{~A}, \mathrm{~B}$ ). On extensive sampling, small foci of squamous epithelial cells were seen intermingled with the neuroectodermal component (Figure 2C). The squamous cells showed dyskeratosis, nuclear hyperchromasia and pleomorphism at places, suggesting malignant transformation. Glandular and ductal structures were not identified. The epithelial islands were surrounded by loose myxoid stroma containing spindle shaped cells showing moderate nuclear pleomorphism and occasional mitotic figures (Figures 2CF). Few cells in the mesenchymal component showed rhabdomyoblastic appearance. An occasional focus of osteoid deposition was also noted. On immunohistochemistry, the neuroectodermal component stained positively with synaptophysin, chromogranin and glial fibrillary acidic protein (Figures 2G, H). The epithelial islands were immunopositive for cytokeratin (Figure 2l) and epithelial membrane antigen (Figure 2J). The stromal cells were diffusely immunopositive for vimentin and focally for desmin, myogenin and smooth muscle actin (Figures 2K-L). There was no evidence of germ cell tumor in any of the sections. Based on the histomorphology and immunohistochemistry, a diagnosis of SNTCS was rendered.

\section{DISCUSSION}

SNTCS is a rare, malignant tumor with complex histomorphology comprising of many components. It is a malignancy of middle-aged adults, with a male preponderance $(4,6)$. It is lo-
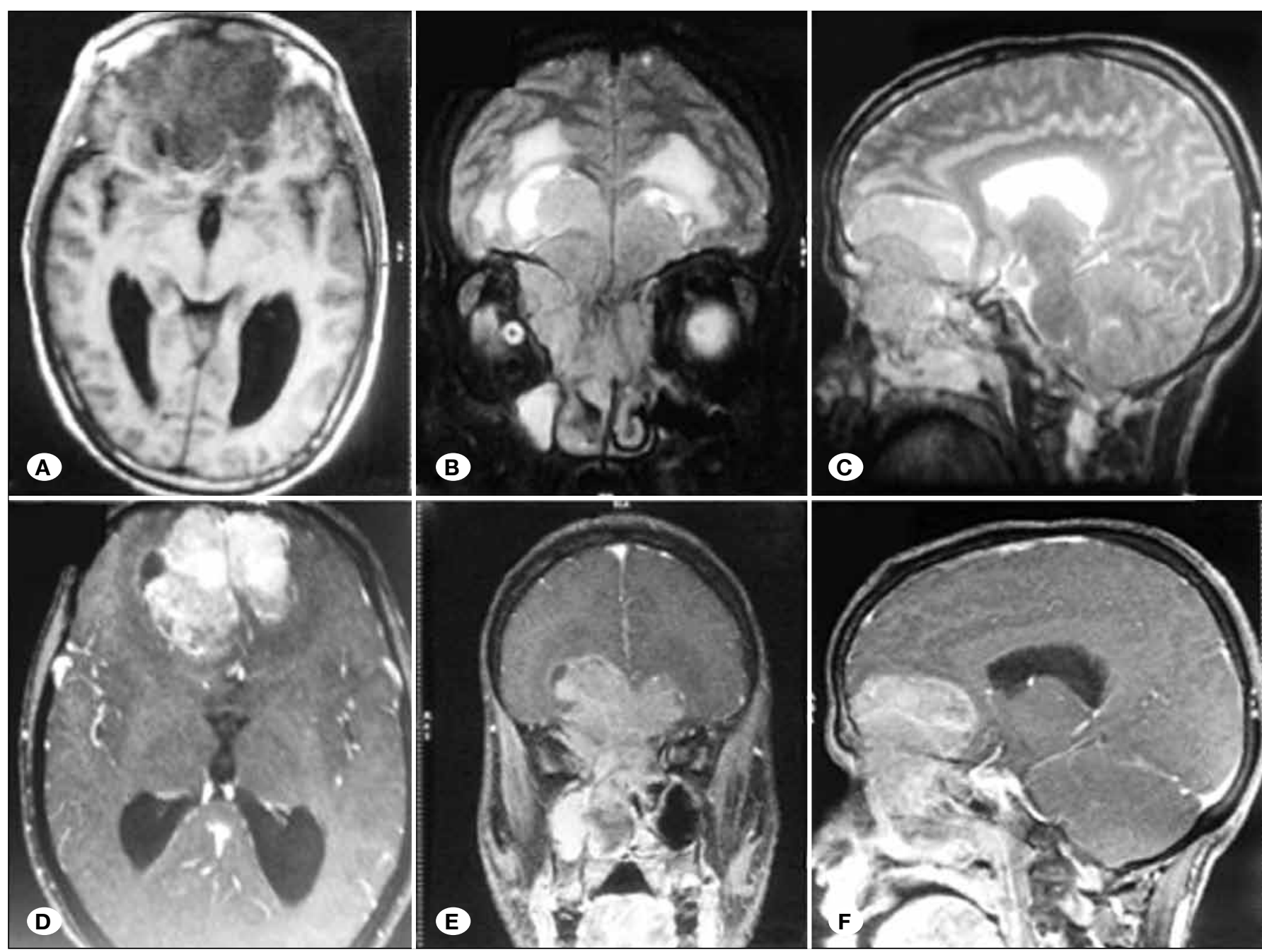

Figure 1: T1-weighted images axial section following hypointense lesion in the anterior skull base (A), T2-weighted images coronal and sagittal sections showing isointense lesion in the anterior skull base and bilateral nasal cavities with extensive perilesional edema (B, C), post contrast images axial, coronal and sagittal sections showing brilliantly and homogenously enhancing lesion in the anterior cranial fossa and bilateral nasal cavities with extension into the right orbit (D-F). 
Sable M. et al: Sinonasal Teratocarcinosarcoma

cated in the nasal cavities, paranasal sinuses, and occasionally at other sites like the orbit, oral cavity, pharynx and anterior cranial fossa (6). The most common presenting symptom is nasal obstruction associated with epistaxis (3). The majority of these tumors show extension into adjacent structures at the time of presentation, and intracranial extension is not uncommon $(3,6)$.

Histologically, SNTCSs show a diversity of tissue types with combination of ectodermal, mesenchymal and endodermal components exhibiting variable degrees of maturity (4). Due to its variegated appearance and midline location, the differential diagnosis of this entity is vast and includes poorly differentiated squamous carcinoma, sarcoma, olfactory neuroblastoma (ONB), malignant craniopharyngioma, small cell carcinoma, sinonasal undifferentiated carcinoma (SNUC), malignant mixed tumor of salivary gland and extragonadal malignant germ cell tumor $(3,8)$. The most commonly identified is the epithelial component, comprising of squamous epithelium and glandular structures. Among these, immature "fetal" type squamous epithelium is most frequent, seen in a significant proportion of cases (4). The malignant counterparts of these epithelial elements are often misdiagnosed as SNUC or squamous cell carcinoma. Similarly, the mesenchymal component may show myofibroblastic cell proliferation with the loose myxoid stroma containing both mature (osteoid, cartilage, smooth muscle or striated muscle) and immature elements, as encountered in the present case. Cytological atypia may range from minimal to complete sarcomatous differentiation. On occasion, the mesenchymal component showing collagenized stroma is erroneously recognized as a desmoplastic response to the carcinomatous component. SNTCS is also frequently associated with neuroectodermal and neuroendocrine differentiation. These small round cells are often misdiagnosed as ONB or neuroendocrine carcinoma (NEC), especially in small biopsies $(1,8)$.

The origin of this tumor remains speculative, although histogenesis from a multipotential adult somatic stem cell with divergent differentiation has been postulated (3). This assumption has been based on the lack of germ cell elements such as those of yolk sac, germinoma, embryonal carcinoma, or choriocarcinoma on histology, and on the absence of demonstrable amplification of $12 p$, most commonly in the form of isochromosome $12 p$ (i12p), which is often associated with tumors of germ cell origin (4).

Although there is no defined treatment protocol, recent reports confirm that multimodality treatment for SNTCS, with maximal safe surgical resection followed by radiotherapy and/

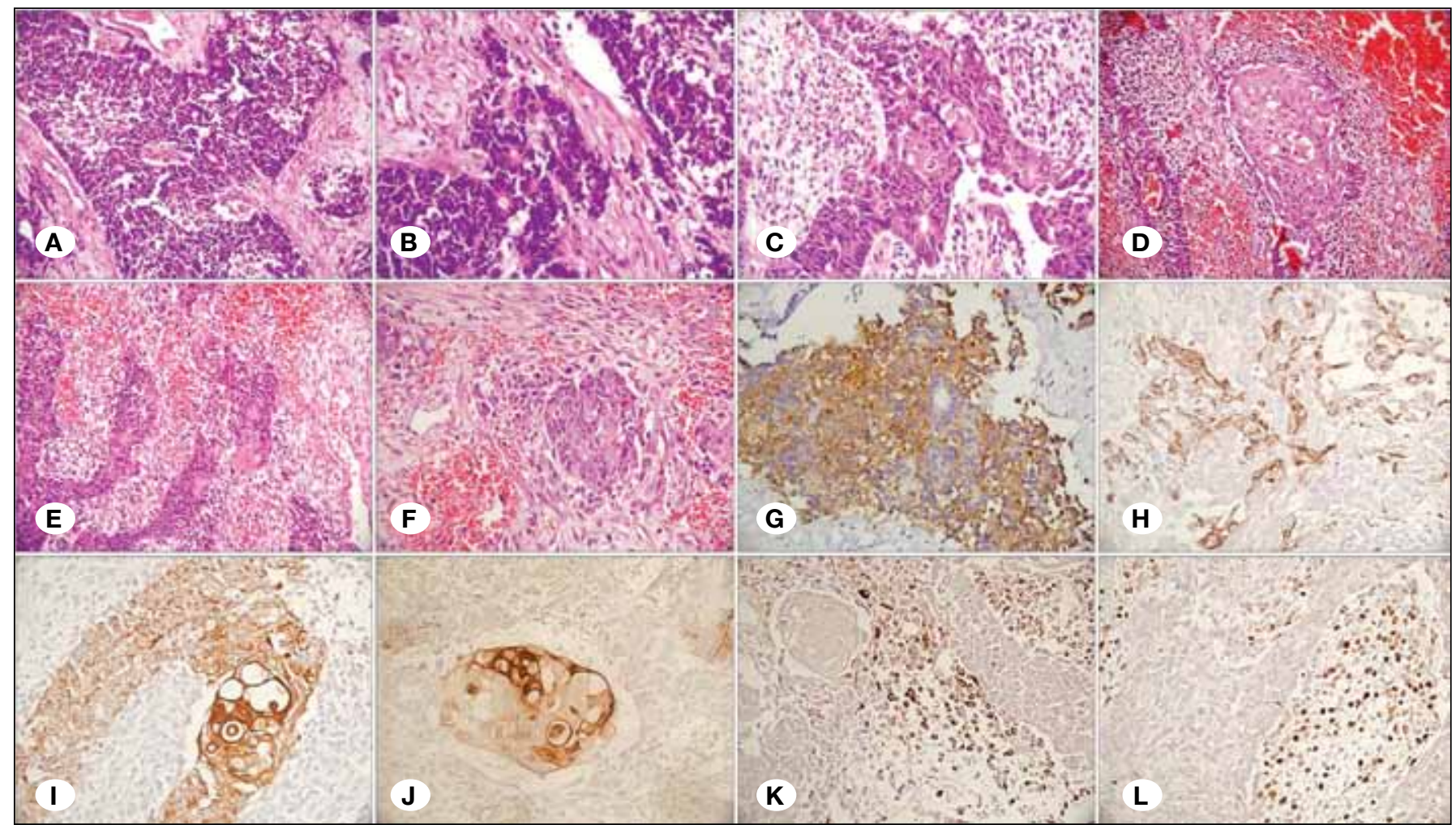

Figure 2: Photomicrograph showing neuroectodermal component - small round cells surrounded by spindle cell stroma (A; HE, $\mathrm{x} 100)$. Higher magnification shows rosettes and myxoid areas in the stroma (B; HE, x200). Small foci of squamous differentiation are seen intermingled with neuroectodermal component (C; HE, x200). Squamous islands are surrounded by polygonal to spindle stromal cells (D; $\mathrm{HE}, \mathrm{x} 200)$ with increased cellularity (E; HE, x200) and mitotic figures (F; HE, x200). There was diffuse immunopositivity for synaptophysin (G; IHC, x100) and focal for GFAP (H; IHC, x200). Epithelial component shows immunopositivity for cytokeratin (I; IHC, x400) and EMA (J; IHC, x400). Stromal cells showed cytoplasmic staining for desmin (K; IHC, x200) and nuclear staining for myogenin at places (I; IHC, $\mathrm{x} 200)$. 
or chemotherapy, is most effective (3). Mean radiotherapy dosage reported in literature is 54.9 Gy to the primary site, while cisplatin is the chemotherapeutic agent used most frequently (3). The outcome of these patients is poor, with reported overall survival rates ranging from $40-46 \%$ at the end of two years $(2,6)$. Majority of patients develop local recurrences, while a few develop lymph node or distant metastases $(6,7)$. A recurrence rate of $37 \%$ and mortality rate of $30 \%$ have been observed in literature (6).

\section{CONCLUSION}

STNCS is a rare, unique entity, which often poses a diagnostic challenge, particularly on small biopsy samples or partially removed tumor specimens. Further, in view of very poor prognosis, multimodality treatment in the form of postoperative radiotherapy and chemotherapy needs to be standardized.

\section{- REFERENCES}

1. Cardesa A, Luna MA: Sinonasal teratocarcinosarcoma. In: Barnes L, Eveson JD, Reichart P, Sidransky D (eds), World Health Organization Classification of Tumours. Pathology and Genetics of Head and Neck Tumours. Lyon: IARC Press, 2005:76-77
2. Heffner DK, Hyams VJ: Teratocarcinosarcoma (malignant teratoma?) of the nasal cavity and paranasal sinuses. A clinicipathological study of 20 cases. Cancer 53:2140-2154, 1984

3. Misra P, Husain Q, Svider PF, Sanghvi S, Liu JK, Eloy JA: Management of sinonasal teratocarcinosarcoma: A systematic review. Am J Otolaryngol 35:5-11, 2014

4. Salem F, Rosenblum MK, Jhanwar SC, Kancherla P, Ghossein RA, Carlson DL: Teratocarcinosarcoma of the nasal cavity and paranasal sinuses: Report of 3 cases with assessment for chromosome 12p status. Hum Pathol 39:605-609, 2008

5. Shanmugaratnam K, Kunaratnam N, Chia KB, Chiang GS, Sinniah R: Teratoid carcinosarcoma of the paranasal sinuses. Pathology 15:413-419, 1983

6. Smith SL, Hessel AC, Luna MA, Malpica A, Rosenthal DI, ElNaggar AK: Sinonasal teratocarcinosarcoma of the head and neck: A report of 10 patients treated at a single institution and comparison with reported series. Arch Otolaryngol Head Neck Surg 134:592-595,2008

7. Wei S, Carroll W, Lazenby A, Bell W, Lopez R, Said-Al-Naief N: Sinonasal teratocarcinosarcoma: Report of a case with review of literature and treatment outcome. Annals of Diagnostic Pathol 12:415-425, 2008

8. Yang S, Sun R, Liang J, Zhou Z, Zhou J, Rui J: Sinonasal teratocarcinosarcoma: A clinical and pathological analysis. Int J Surg Pathol 21:37-43, 2013 